\title{
The association between EGFR variant III, HPV, p16, c-MET, EGFR gene copy number and response to EGFR inhibitors in patients with recurrent or metastatic squamous cell carcinoma of the head and neck
}

\author{
Nicole G Chau', Bayardo Perez-Ordonez ${ }^{2}$, Katherine Zhang ${ }^{3}$, Nhu-An Pham ${ }^{3}$, James Ho ${ }^{3}$, Tong Zhang ${ }^{3}$, \\ Olga Ludkovski ${ }^{3}$, Lisa Wang ${ }^{4}$, Eric X Chen ${ }^{1}$, Ming-Sound Tsao ${ }^{3}$, Suzanne Kamel-Reid ${ }^{3}$, Lillian L Siu ${ }^{1 *}$
}

\begin{abstract}
Background: We examine the potential prognostic and predictive roles of EGFR variant III mutation, EGFR gene copy number (GCN), human papillomavirus (HPV) infection, c-MET and p16 $6^{\text {INKAA }}$ protein expression in recurrent or metastatic squamous cell carcinoma of the head and neck (R/M SCCHN).

Methods: We analyzed the archival tumor specimens of 53 patients who were treated in 4 phase II trials for R/M SCCHN. Two trials involved the EGFR inhibitor erlotinib, and 2 trials involved non-EGFR targeted agents. EGFRvIll mutation was determined by quantitative RT-PCR, HPV DNA by Linear Array Genotyping, p16 and c-MET protein expression by immunohistochemistry, and EGFR GCN by FISH.

Results: EGFRvIll mutation, detected in 22 patients (42\%), was associated with better disease control, but no difference was seen between erlotinib-treated versus non-erlotinib treated patients. EGFRvIll was not associated with TTP or OS. The presence of HPV DNA (38\%), p16 immunostaining (32\%), c-MET high expression (58\%) and EGFR amplification (27\%), were not associated with response, TTP or OS.
\end{abstract}

Conclusion: EGFRvIll mutation, present in about 40\% of SCCHN, appears to be an unexpected prognostic biomarker associated with better disease control in R/M SCCHN regardless of treatment with erlotinib. Larger prospective studies are required to validate its significance.

\section{Background}

The epidermal growth factor receptor (EGFR) is overexpressed in up to $90 \%$ of squamous cell carcinoma of the head and neck (SCCHN) and has been postulated to be a key molecular target in this malignancy [1]. EGFR signal transduction leads to cell proliferation, invasion, angiogenesis and metastasis [2]. EGFR overexpression and aberrant EGFR gene copy number (EGFR GCN) have been associated with poorer prognosis and diseasespecific survival in SCCHN $[1,3,4]$. Therapies targeted

\footnotetext{
* Correspondence: lillian.siu@uhn.on.ca

'Division of Medical Oncology and Hematology, Princess Margaret Hospital, University Health Network, Toronto, Ontario, Canada

Full list of author information is available at the end of the article
}

against EGFR have demonstrated modest activity either alone or in combination with chemotherapy in both locally advanced [5] and recurrent and/or metastatic SCCHN [6-10]. No validated biomarkers exist to predict the response to EGFR inhibitors in SCCHN.

The most common EGFR truncation mutation, EGFR variant III (EGFRvIII), harbors an in-frame deletion of exons 2 to 7 (801 bp), resulting in a truncated extracellular EGF-binding domain that is constitutively activated and ineffectively ubiquinated $[11,12]$. EGFRvIII is found in many human cancers and is present in $\sim 40 \%$ of glioblastomas and $5 \%$ of lung squamous cell carcinomas, where it confers tumorigenicity and dose-dependent resistance to gefitinib in pre-clinical models $[13,14]$. The 
prevalence of EGFRvIII in SCCHN was first reported as $43 \%$ in one study of 33 SCCHN tumors [15]. EGFRvIIItransfected SCCHN cells had decreased apoptosis in response to cisplatin and decreased growth inhibition following treatment with the EGFR monoclonal antibody cetuximab compared with controls [15]. EGFRvIII is an interesting therapeutic target because unlike wild-type EGFR, EGFRvIII is not found in normal tissue. EGFRvIII is proposed to account for limitations in response to current EGFR inhibitors, however in patients with SCCHN tumors harboring EGFRvIII response to EGFR tyrosine kinase inhibition (TKI) is unknown.

$\mathrm{HPV}$ infection is a risk factor for the development of SCCHN. HPV DNA is found in $20-30 \%$ of SCCHN and up to $40-66 \%$ of SCCHN of the oropharynx $[16,17]$. HPV positive oropharyngeal tumors are clinically and molecularly distinct from HPV negative tumors $[18,19]$ and associated with a more favorable prognosis [20]. HPV positive status prospectively predicts survival and response to induction chemotherapy and chemoradiation in stage III or IV oropharynx cancers [21,22] and better response to radiotherapy alone [23]. The combination of low HPV titers and high EGFR expression was associated with worse overall survival in oropharynx cancer [22]. Inactivation of pRb by HPV E7 protein results in overexpression of $\mathrm{p} 16$ protein, thus $\mathrm{p} 16$ immunostaining has served as a surrogate marker for HPV-associated SCCHN. Patients with tumors lacking both p16 expression and HPV (p16-/HPV-) had the worst disease-specific survival compared to tumors with p16+/HPV+, p16-/HPV+ or p16+/HPV- types [24]. Despite the importance of HPV in the pathogenesis and prognosis of SCCHN in response to chemotherapy and radiation, the role of HPV DNA and response to EGFR inhibitors in SCCHN is unclear.

c-MET, a proto-oncogene tyrosine kinase receptor, is overexpressed in SCCHN, and its ligand, hepatocyte growth factor (HGF), stimulates cell proliferation, motility and invasion [25]. c-MET overexpression has been associated with disease progression in oral squamous cell carcinoma (OSCC) [26]. Elevated serum HGF is associated with resistance to chemoradiation and reduced survival [27]. c-MET amplification and mutations of MET confer an invasive phenotype associated with metastases in SCCHN [28]. Ligand-independent constitutive activation of c-MET via its heterodimerization with EGFR has been identified as a contributing mechanism of acquired resistance to cetuximab in SCCHN [29]. The role of c-MET in response to EGFR TKI in the clinical setting in SCCHN is unknown.

In this study, we examine the prevalence of EGFRvIII, HPV, p16, c-MET and EGFR GCN in patients with R/M SCCHN and explore the potential prognostic and predictive roles of these biomarkers in patients treated with or without EGFR TKI. We hypothesized that EGFRvIII and $\mathrm{C}-\mathrm{MET}$ would be associated with poorer prognosis or response to EGFR TKI, while HPV and p16 expression would predict improved clinical outcomes and response to treatment.

\section{Methods}

\section{Patients}

We obtained approval from the University Health Network Research Ethics Board to evaluate the archival formalin-fixed paraffin embedded (FFPE) tumor specimens of patients with $\mathrm{R} / \mathrm{M}$ SCCHN who were treated in four phase II trials for R/M SCCHN at Princess Margaret Hospital conducted from 2000-2005. Two of the four trials involved the EGFR TKI erlotinib (phase II trial of erlotinib [8], phase II trial of erlotinib and cisplatin [7]) and the remaining two trials used other non-EGFR targeted agents (phase II trial of the kinesin spindle protein inhibitor ispinesib [30], phase II trial of the multi-kinase antiangiogenic inhibitor sorafenib [31]). The medical records and case report forms were reviewed to obtain patient demographics, primary tumor site, treatment details and clinical outcome (response rate, time to progression and overall survival).

\section{Specimen Characteristics}

Archival FFPE tumor specimens were available in 35 of 48 patients $(73 \%)$ treated with erlotinib and 18 of 37 (49\%) patients treated with non-EGFR targeted agents. H\&E stained sections were examined by a histopathologist (B.P-O.) to confirm the presence of $>80 \%$ tumor in the specimens evaluated.

\section{Assay Methods/Molecular Assays \\ EGFRvIII Mutation Detection}

RNA Isolation RNA was isolated in tumor area on the FFPE slides guided by H\&E-stained serial sections. The tissues were deparaffinized by xylene and ethanol. Total RNA from paraffin-embedded tissues was extracted using RecoveryAll ${ }^{\mathrm{TM}}$ Total Nucleic Acid isolation Kit (Ambion Diagnostics, Streetsville, Ontario, Canada).

Real-time $\boldsymbol{R}$ T-PCR Reverse transcription was done using TaqMan Reverse transcription reagent kit (Roche, Branchburg, New Jersey) according to the manufacturer's protocol. Reverse transcription reaction was done in a total volume of $25 \mathrm{~mL}$ including RNA template, $1.25 \mathrm{~mL}$ random hexamer, reverse transcription buffer, $5.0 \mathrm{~mL}$ dNTP, $5.5 \mathrm{~mL} \mathrm{MgCl}$, RNase inhibitor, and M-MLV reverse transcriptase.

Real-time PCR was performed in duplicate in $25 \mathrm{~mL}$ reaction volumes using Platinum SYBR Green qPCR SuperMix-UDG (Invitrogen, Carlsbad, California) and a 7900HT instrument (Applied Biosystems, Foster City, California). The amplification conditions were: $50^{\circ} \mathrm{C}$ for 
$2 \mathrm{~min}$., $95^{\circ} \mathrm{C}$ for $2 \mathrm{~min}$., 40 cycles of $95^{\circ} \mathrm{C}$ for $15 \mathrm{sec}$. and $60^{\circ} \mathrm{C}$ for $1 \mathrm{~min}$.

Data Analysis of real-time PCR A mixture of at least eight normal FFPE tissue samples was used as a wildtype, normal control. The EGFRvlll cell line (u373flagEGFRvlll) was used as a positive control.

The relative expression of EGFR exon 4 to EGFR exon 9 was determined using the delta delta $\mathrm{Ct}(\Delta \Delta \mathrm{Ct})$ method. All samples were run in duplicate, and the mean $\mathrm{Ct}$ number was used for data analysis. The difference in $\mathrm{Ct}$ values ( $\Delta$ threshold cycle, $\Delta \mathrm{Ct}=$ Exon $9 \mathrm{Ct}$ Exon $4 \mathrm{Ct}$ ) was calculated for each RNA sample. The $\Delta \mathrm{Ct}$ from the normal tissue mixture was then subtracted from the $\Delta \mathrm{Ct}$ of the test sample to generate a $\Delta \Delta \mathrm{Ct}$. A negative result occurs when the fold change (exon 9: exon 4 calculated as $2^{-\Delta \Delta C t}$ ) is less than a value of 5 . This value was arbitrarily chosen to ensure that no false positives were called. A positive result occurs when the fold change is the same as or greater than that of the positive control (7). When the fold change of tested samples falls between that of the normal control and the positive control (i.e. between 5 and 7) the results are considered inconclusive.

\section{HPV DNA Detection}

The Roche Linear Array HPV Genotyping kit (Roche Molecular Diagnostics, Pleasanton, California) was used to detect 37 low- and high-risk HPV types from FFPE tissues. In brief, FFPE sections were deparaffinized and DNA was extracted using a column based method (QIAamp, Qiagen, Valencia, California). HPV detection was performed using PCR amplification followed by hybridization of the amplified products to oligonucleotide probes and subsequent colorimetric determination. All experiments included an HPV positive control and an HPV negative control.

HPV DNA by in situ hybridization (ISH) using the INFORM HPV III Family 16 probe (Ventana Medical Systems Inc., Tucson, Arizona) which detects genotypes $16,18,31,33,39,35,45,51,52,56,58$ and 66 , was performed according to the manufacturer's guidelines using the Ventana Benchmark automated slide staining system. All experiments included an HPV positive control and an HPV negative control. Slides were scored as positive if a punctate or diffuse pattern of signal were observed in the tumor nuclei.

\section{P16 and c-MET Detection}

Immunohistochemistry (IHC) for p16 and c-MET using the Ventana Benchmark XT auto-immunostainer (Tucson, Arizona) was performed on FFPE sections cut at $4 \mathrm{~mm}$ thick. Standardized staining protocols were provided by Ventana for the CINtec p16 Histology kit (MTM Laboratories Inc, Westborough Massachusetts) and c-MET antibody (SP44, rabbit monoclonal, Ventana Medical Systems Inc., Tucson Arizona). Controls were included in each assay, comprising of positive tissue controls and negative controls. All p16 and c-MET IHC slides were reviewed independently by two observers (B.P.O. and N.G.C.) without knowledge of EGFRvIII, HPV status or clinical outcome. p16 staining in SCCHN is generally observed to be dichotomous and scored as absent (weak or no staining) or present (strong and diffuse staining) [32]. c-MET IHC slides were assigned a semi-quantitative score based on the product of an intensity score $(0=$ no staining or equal to background, $1=$ weak, or $2=$ strong) and percent of area stained $(0=0 \%, 1=1-30 \%, 2=31-60 \%, 3=>60 \%)$. Sections with an inter-observer variation were reassessed by a double-headed light microscope to achieve consensus.

\section{EGFR Gene Copy Number}

Archival tumor specimens were analyzed for EGFR GCN using fluorescent in situ hybridization (FISH) as previously described [33,34]. One hundred non-overlapping interphase nuclei were scored for EGFR and CEP7 copy number and classified into six categories (University of Colorado Scoring system) by a reviewer blinded to clinical outcome (O.L.) [35].

\section{Statistical Methods}

Descriptive statistics were used to summarize the study cohort and to estimate the parameters of interest. Ninety-five percent confidence intervals were obtained for estimates of the presence of EGFRvIII, HPV, p16, c-MET and EGFR GCN. Exploratory analyses were performed to characterize the relationships between EGFRvIII, HPV, p16, c-MET and EGFR GCN with baseline patient characteristics and outcomes. Only patients with conclusive EGFRvIII results were included in the correlation analyses. The Kaplan-Meier method was used to estimate the overall survival and time to progression. All biomarkers were examined in univariate analysis of overall survival and time to progression using Cox proportional hazards model. Only those which were significant at 0.10 (two-sided) level in the univariate analysis were entered in the multivariate analysis and markers that remained significant at 0.05 (two-sided) level in the multivariate analysis were considered significant prognostic factors. Statistical analyses were performed using the SAS 9.1 software package (SAS Institute, Cary, North Carolina).

\section{Results}

\section{Patients}

The clinical characteristics of the 53 patients in our study are described in Table 1. For the entire cohort, the overall response rate $(\mathrm{CR}+\mathrm{PR})$ to study treatment was $4 / 53(7.5 \%)$, median time to progression (TTP) was 1.8 months (95\% CI 1.6-2.7) and median overall survival (OS) was 5.9 months (95\% CI 4.5-8.7). Patients in the 
Table 1 Clinical characteristics of the entire study cohort ( $n=53$ )

\begin{tabular}{|c|c|c|}
\hline \multicolumn{2}{|l|}{ Clinical Characteristic } & \multirow{2}{*}{$\begin{array}{l}\text { Number } \\
56(15-78 \\
\end{array}$} \\
\hline Median Age (Range) & & \\
\hline Gender & Female:Male & $12: 41$ \\
\hline ECOG Performance Status & $0: 1: 2$ & $15: 34: 4$ \\
\hline Locoregional Recurrence & Yes:No & $45: 8$ \\
\hline Distant Metastases & Yes:No & 19:34 \\
\hline \multirow[t]{6}{*}{ Primary Tumor Site } & Oropharynx & 20 \\
\hline & Larynx & 14 \\
\hline & Oral cavity & 10 \\
\hline & Hypopharynx & 2 \\
\hline & Neck mass unknown & 4 \\
\hline & Paranasal sinus & 3 \\
\hline \multirow[t]{4}{*}{ Histologic Grade } & Well differentiated & 5 \\
\hline & Moderately differentiated & 33 \\
\hline & Poorly differentiated & 14 \\
\hline & Infiltrating basaloid & 1 \\
\hline \multirow[t]{3}{*}{ Prior Therapy } & Chemotherapy & 21 \\
\hline & Radiation Therapy & 51 \\
\hline & Surgery & 41 \\
\hline \multirow[t]{4}{*}{ Race } & Asian & 8 \\
\hline & Black & 42 \\
\hline & Caucasian & 9 \\
\hline & Other & 1 \\
\hline \multirow[t]{4}{*}{ Smoker } & Current & 33 \\
\hline & Former & 3 \\
\hline & Never & 15 \\
\hline & Unknown & 2 \\
\hline Erlotinib & Yes: No & $35: 18$ \\
\hline \multirow[t]{4}{*}{ Best Response } & Partial response & 4 \\
\hline & Stable disease & 20 \\
\hline & Progressive disease & 24 \\
\hline & Inevaluable & 5 \\
\hline
\end{tabular}

erlotinib group had a higher median OS of 7.9 months (95\% CI 4.7-9.8) compared to patients in the nonerlotinib group with median OS of 4.2 months (95\% CI $2.9-7.0)(\mathrm{p}=0.011)$. The erlotinib group had a higher TTP than the non-erlotinib group 2.7 months (95\% CI 1.6-3.5) vs 1.5 months (95\% CI 1.3-1.8) ( $\mathrm{p}=0.0009$ ).

\section{Expression of EGFRvIII mutation by real-time $P C R$}

As the previously reported immunohistochemistry-suitable antibody [15] against EGFRvIII is no longer available, EGFRvIII expression is analysed using the RT-PCR method. The presence of EGFRvIII mutation was detected in 22 patients (42\%) (Table 2), negative in 19 patients and inconclusive in 12 patients (Table 3). The median EGFRvIII fold change was 6.8 (0.56 to 576.36$)$ for all patients, 15.0 (4.1 to 576.36 ) for patients in the EGFRvIII positive group, 1.8 (0.6 to 4.3) for patients in the EGFRvIII negative group, and 6.5 (6.2 to 6.8 ) for patients in the inconclusive group.

Patients with tumors harboring the EGFRvIII mutation had similar clinical characteristics to patients without the EGFRvIII mutation (Table 4).

EGFRvIII is associated with disease control In univariate analysis, the presence of EGFRvIII was associated with better disease control (Table 5). Median EGFRvIII fold changes were higher for patients with disease control than patients with progressive disease (11.11 vs. $3.16, \mathrm{p}=0.04)$. No significant difference was observed between erlotinib-treated ( $\mathrm{p}=0.21$ ) versus non-erlotinib $(\mathrm{p}=0.10)$ treated patients due to the small sample size (Table 5). The presence of EGFRvIII mutation was not associated with TTP (HR 0.94 (95\% CI 0.33-2.71), p = 0.91 ) or OS (HR 0.91 (95\% CI 0.32-2.60), $\mathrm{p}=0.85$ ) (Figure 1).

HPV DNA

HPV DNA testing by PCR was positive in 20 patients (38\%), negative in 31 patients and inconclusive in 2 patients (Tables 2 and 3). The most prevalent HPV subtype found in our analysis was the high-risk HPV-16 (18/20 cases). The majority of HPV-16 positive tumors were from the oropharynx (12/18). HPV positive tumor status was not significantly associated with disease control (Table 6), TTP (HR 1.19 (95\% CI 0.46-3.11), p = 0.722 ) or OS (HR 0.88 (95\% CI 0.34-2.29), $\mathrm{p}=0.788$ ).

\section{P16}

P16 immunoreactivity was detected in 17 patients (32\%), absent in 33 patients and inconclusive in 3 patients (Tables 2 and 3). The inter-observer variability rate was $6 \%$ and discrepant cases were resolved by consensus review. P16 expression was not associated with disease control (Table 6), TTP (HR 0.50 (95\% CI 0.19-1.32), p = 0.16 ) or OS (HR 0.61 (95\%CI 0.24-1.55), $\mathrm{p}=0.30$ ).

The discordance between p16 IHC and HPV DNA by PCR was 25\% (Table 7). To investigate this further, we performed HPV DNA by ISH. The discordance between p16 IHC and HPV DNA by ISH was lower at $16 \%$ (Table 7) and all 7 discordant cases were p16-positive/ HPV-ISH-negative. Of these 7 discordant cases, 2 cases were HPV-16 positive by PCR, 4 cases were HPV negative by PCR and 1 case was inconclusive by HPV PCR. C-MET

Forty-nine patients had sufficient tumor samples for evaluation of c-MET. Eighteen patients (63\%) had low c-MET scores of 0,1 or 2 and 31 patients had high c-MET (>2) (Tables 2 and 3). Less than 10\% interobserver variability was observed and discrepant cases were resolved by consensus review. High c-MET was not associated with disease control (Table 6), TTP (HR 1.47 (95\%CI $0.56-3.85), \mathrm{p}=0.43)$ or OS (HR $1.72(95 \%$ CI $0.65-4.56), \mathrm{p}=0.27)$. 
Table 2 EGFRvIII mutation positive detected by RT-PCR $(n=22)$

\begin{tabular}{|c|c|c|c|c|c|c|c|c|c|}
\hline Case & Treatment & Primary Site & Specimen Site & $\begin{array}{l}\text { EGFRvIll by } \\
\text { RT-PCR }\end{array}$ & $\begin{array}{l}\text { EGFRvill Fold } \\
\text { Changes }\end{array}$ & $\begin{array}{l}\text { HPV DNA by } \\
\text { Linear Array }\end{array}$ & $\begin{array}{l}\text { P16 } \\
\text { IHC }\end{array}$ & $\begin{array}{l}\text { MET score } \\
\text { by IHC }\end{array}$ & EGFR FISH \\
\hline 1 & Erlotinib & hypopharynx & $\begin{array}{l}\text { Untreated } \\
\text { primary }\end{array}$ & + & 11.33 & - & - & High & $\begin{array}{l}\text { Low } \\
\text { polysomy }\end{array}$ \\
\hline 2 & Erlotinib & oral cavity & $\begin{array}{l}\text { Untreated } \\
\text { primary }\end{array}$ & + & 7.01 & - & - & High & Disomy \\
\hline 3 & Erlotinib & larynx & $\begin{array}{c}\text { Untreated } \\
\text { primary }\end{array}$ & + & 61.77 & - & - & Low & $\begin{array}{c}\text { Low } \\
\text { polysomy }\end{array}$ \\
\hline 4 & Erlotinib & larynx & $\begin{array}{l}\text { Local } \\
\text { recurrence }\end{array}$ & + & 26.64 & - & - & Low & $\begin{array}{c}\text { High } \\
\text { polysomy }\end{array}$ \\
\hline 5 & Erlotinib & $\begin{array}{c}\text { neck mass } \\
\text { unknown primary }\end{array}$ & $\begin{array}{l}\text { Untreated } \\
\text { lymph node }\end{array}$ & + & 60.04 & $33+$ & + & High & Low trisomy \\
\hline 6 & Erlotinib & oral cavity & $\begin{array}{l}\text { Untreated } \\
\text { lymph node }\end{array}$ & + & 218.49 & - & - & High & Amplification \\
\hline 7 & Erlotinib & oropharynx & $\begin{array}{l}\text { Unknown } \\
\text { primary }\end{array}$ & + & 15.66 & $16+$ & + & High & Low trisomy \\
\hline 8 & Erlotinib & oral cavity & $\begin{array}{l}\text { Local } \\
\text { recurrence }\end{array}$ & + & 8.94 & - & - & High & $\begin{array}{c}\text { Low } \\
\text { polysomy }\end{array}$ \\
\hline 9 & $\begin{array}{c}\text { Erlotinib + } \\
\text { Cisplatin }\end{array}$ & $\begin{array}{c}\text { neck mass } \\
\text { unknown primary }\end{array}$ & $\begin{array}{l}\text { Untreated } \\
\text { lymph node }\end{array}$ & + & 127.84 & $16+$ & $\mathrm{NE}$ & NE & Low trisomy \\
\hline 10 & $\begin{array}{c}\text { Erlotinib + } \\
\text { Cisplatin }\end{array}$ & larynx & $\begin{array}{c}\text { Node } \\
\text { recurrence }\end{array}$ & + & 576.36 & $16+$ & + & High & Disomy \\
\hline 11 & $\begin{array}{c}\text { Erlotinib + } \\
\text { Cisplatin }\end{array}$ & larynx & $\begin{array}{l}\text { Untreated } \\
\text { primary }\end{array}$ & + & 8.26 & - & - & Low & Failed \\
\hline 12 & $\begin{array}{c}\text { Erlotinib + } \\
\text { Cisplatin }\end{array}$ & larynx & $\begin{array}{l}\text { Untreated } \\
\text { primary }\end{array}$ & + & 17.38 & - & - & High & $\begin{array}{c}\text { Low } \\
\text { polysomy }\end{array}$ \\
\hline 13 & $\begin{array}{l}\text { Erlotinib + } \\
\text { Cisplatin }\end{array}$ & oropharynx & $\begin{array}{l}\text { Untreated } \\
\text { primary }\end{array}$ & + & 14.28 & $16+$ & + & High & Disomy \\
\hline 14 & $\begin{array}{l}\text { Erlotinib + } \\
\text { Cisplatin }\end{array}$ & oral cavity & $\begin{array}{l}\text { Untreated } \\
\text { primary }\end{array}$ & + & 69.68 & - & NE & NE & $\begin{array}{c}\text { Low } \\
\text { polysomy }\end{array}$ \\
\hline 15 & $\begin{array}{c}\text { Erlotinib + } \\
\text { Cisplatin }\end{array}$ & oral cavity & $\begin{array}{c}\text { Untreated } \\
\text { primary }\end{array}$ & + & 11.11 & $53+, 58+, 6+, 52+$ & - & High & $\begin{array}{l}\text { Low } \\
\text { polysomy }\end{array}$ \\
\hline 16 & Sorafenib & oropharynx & $\begin{array}{l}\text { Untreated } \\
\text { primary }\end{array}$ & + & 7.71 & $16+$ & + & Low & $\begin{array}{c}\text { Low } \\
\text { polysomy }\end{array}$ \\
\hline 17 & Sorafenib & oropharynx & $\begin{array}{l}\text { Untreated } \\
\text { primary }\end{array}$ & + & 7.93 & $16+$ & + & Low & Low trisomy \\
\hline 18 & Ispinesib & $\begin{array}{c}\text { neck mass } \\
\text { unknown primary }\end{array}$ & $\begin{array}{l}\text { Untreated } \\
\text { lymph node }\end{array}$ & + & 4.12 & $16+, 53+, 51+$ & + & High & $\begin{array}{l}\text { High } \\
\text { polysomy }\end{array}$ \\
\hline 19 & Ispinesib & oropharynx & $\begin{array}{l}\text { Untreated } \\
\text { primary }\end{array}$ & + & 15.61 & $\begin{array}{c}16+, 53+, 33+, 51 \\
+, 58+\end{array}$ & - & High & $\begin{array}{l}\text { High } \\
\text { polysomy }\end{array}$ \\
\hline 20 & Ispinesib & larynx & $\begin{array}{l}\text { Untreated } \\
\text { primary }\end{array}$ & + & 218.26 & - & - & High & Disomy \\
\hline 21 & Ispinesib & oropharynx & $\begin{array}{l}\text { Local } \\
\text { recurrence }\end{array}$ & + & 29.25 & $16+, 53+, 51+$ & + & Low & Disomy \\
\hline 22 & Ispinesib & hypopharynx & $\begin{array}{l}\text { Local } \\
\text { recurrence }\end{array}$ & + & 9.31 & $\begin{array}{c}16+, 53+, 58+, 52 \\
+\end{array}$ & - & High & $\begin{array}{l}\text { Low } \\
\text { polysomy }\end{array}$ \\
\hline
\end{tabular}

Abbreviations: +, positive; -, negative; $\mathrm{NE}$, not evaluable.

EGFR Gene Copy Number

Forty-five patients had sufficient tumor samples for evaluation of EGFR GCN by FISH. High EGFR GCN (amplification and high polysomy) was detected in 13 patients and low EGFR GCN (disomy, low polysomy) was detected in 33 patients (Tables 2 and 3). High EGFR GCN was not predictive for TTP (HR 0.99, p = 0.822 ) or OS (HR 1.10, $\mathrm{p}=0.644)$. High EGFR GCN was not associated with the presence of EGFRvIII ( $\mathrm{p}=$ 0.14 Fisher's exact test).

\section{Discussion}

To the best of our knowledge, this is the first study to evaluate the role of EGFRvIII in a cohort of patients with R/M SCCHN treated with or without EGFR TKI. This study confirms that EGFRvIII mutation is common 
Table 3 EGFRvIII mutation negative detected by RT-PCR $(n=19)$ and inconclusive cases $(n=12)$

\begin{tabular}{|c|c|c|c|c|c|c|c|c|c|}
\hline Case & Treatment & Primary Site & $\begin{array}{l}\text { Specimen } \\
\text { Site }\end{array}$ & $\begin{array}{l}\text { EGFRvill by } \\
\text { RT-PCR }\end{array}$ & $\begin{array}{l}\text { EGFRvill Fold } \\
\text { Changes }\end{array}$ & $\begin{array}{l}\text { HPV DNA by } \\
\text { Linear Array }\end{array}$ & $\begin{array}{l}\text { P16 } \\
\text { IHC }\end{array}$ & $\begin{array}{l}\text { MET score } \\
\text { by IHC }\end{array}$ & EGFR FISH \\
\hline 23 & Erlotinib & larynx & $\begin{array}{l}\text { Local } \\
\text { recurrence }\end{array}$ & - & 1.76 & - & - & High & Low polysomy \\
\hline 24 & Erlotinib & larynx & $\begin{array}{l}\text { Untreated } \\
\text { primary }\end{array}$ & - & 0.95 & - & - & $\mathrm{NE}$ & $\mathrm{NE}$ \\
\hline 25 & Erlotinib & oropharynx & $\begin{array}{l}\text { Untreated } \\
\text { primary }\end{array}$ & Incon. & $\mathrm{NE}$ & $16+$ & - & Low & Failed \\
\hline 26 & Erlotinib & larynx & Untreated & Incon. & $\mathrm{NE}$ & - & - & $\mathrm{NE}$ & $\mathrm{NE}$ \\
\hline 27 & Erlotinib & oropharynx & $\begin{array}{l}\text { Local } \\
\text { recurrence }\end{array}$ & - & 2.56 & $16+$ & - & High & Failed \\
\hline 28 & Erlotinib & $\begin{array}{c}\text { neck mass } \\
\text { unknown primary }\end{array}$ & $\begin{array}{l}\text { Local } \\
\text { recurrence }\end{array}$ & - & 1.81 & - & - & High & Low polysomy \\
\hline 29 & Erlotinib & paranasal sinus & $\begin{array}{l}\text { Untreated } \\
\text { primary }\end{array}$ & - & 0.56 & - & - & High & High polysomy \\
\hline 30 & Erlotinib & oropharynx & $\begin{array}{l}\text { Untreated } \\
\text { primary }\end{array}$ & - & 3.16 & $16+$ & + & High & Low trisomy \\
\hline 31 & Erlotinib & larynx & $\begin{array}{l}\text { Untreated } \\
\text { primary }\end{array}$ & - & 3.17 & - & - & High & Low polysomy \\
\hline 32 & Erlotinib & oropharynx & $\begin{array}{l}\text { Local } \\
\text { recurrence }\end{array}$ & $\mathrm{NE}$ & $\mathrm{NE}$ & - & + & Low & Low polysomy \\
\hline 33 & Erlotinib & larynx & $\begin{array}{l}\text { Untreated } \\
\text { primary }\end{array}$ & - & 3.16 & - & - & High & High trisomy \\
\hline 34 & $\begin{array}{c}\text { Erlotinib + } \\
\text { Cisplatin }\end{array}$ & oropharynx & $\begin{array}{l}\text { Untreated } \\
\text { primary }\end{array}$ & Incon. & NE & Incon. & + & Low & NE \\
\hline 35 & $\begin{array}{l}\text { Erlotinib + } \\
\text { Cisplatin }\end{array}$ & larynx & $\begin{array}{l}\text { Untreated } \\
\text { primary }\end{array}$ & - & 4.29 & - & - & Low & Low polysomy \\
\hline 36 & $\begin{array}{c}\text { Erlotinib + } \\
\text { Cisplatin }\end{array}$ & oral cavity & $\begin{array}{l}\text { Untreated } \\
\text { primary }\end{array}$ & - & 0.9 & - & - & Low & High polysomy \\
\hline 37 & $\begin{array}{l}\text { Erlotinib + } \\
\text { Cisplatin }\end{array}$ & paransal sinus & $\begin{array}{l}\text { Local } \\
\text { recurrence }\end{array}$ & Incon. & $\mathrm{NE}$ & $16+, 53+$ & Incon. & Low & Low polysomy \\
\hline 38 & $\begin{array}{l}\text { Erlotinib + } \\
\text { Cisplatin }\end{array}$ & larynx & $\begin{array}{l}\text { Local } \\
\text { recurrence }\end{array}$ & Incon. & $\mathrm{NE}$ & $\begin{array}{c}16+, 53+, 33+, 51 \\
+\end{array}$ & + & High & Disomy \\
\hline 39 & $\begin{array}{c}\text { Erlotinib + } \\
\text { Cisplatin }\end{array}$ & paranasal sinus & $\begin{array}{l}\text { Local } \\
\text { recurrence }\end{array}$ & - & 2.59 & $6+$ & - & High & NE \\
\hline 40 & $\begin{array}{c}\text { Erlotinib + } \\
\text { Cisplatin }\end{array}$ & oral cavity & $\begin{array}{l}\text { Untreated } \\
\text { primary }\end{array}$ & - & 2.01 & - & - & High & Disomy \\
\hline 41 & $\begin{array}{c}\text { Erlotinib + } \\
\text { Cisplatin }\end{array}$ & oropharynx & $\begin{array}{c}\text { Untreated } \\
\text { primary }\end{array}$ & Incon. & $\mathrm{NE}$ & - & + & High & Low trisomy \\
\hline 42 & $\begin{array}{c}\text { Erlotinib + } \\
\text { Cisplatin }\end{array}$ & oral cavity & $\begin{array}{l}\text { Untreated } \\
\text { primary }\end{array}$ & Incon. & $\mathrm{NE}$ & - & - & High & High polysomy \\
\hline 43 & Sorafenib & oral cavity & $\begin{array}{l}\text { Untreated } \\
\text { primary }\end{array}$ & Incon. & 6.8 & - & - & Low & Low polysomy \\
\hline 44 & Sorafenib & oropharynx & $\begin{array}{l}\text { Untreated } \\
\text { primary }\end{array}$ & - & 0.99 & - & + & High & $\begin{array}{l}\text { Amplification/ } \\
\text { High trisomy }\end{array}$ \\
\hline 45 & Sorafenib & oral cavity & $\begin{array}{l}\text { Local } \\
\text { recurrence }\end{array}$ & Incon. & NE & Incon. & - & Low & Low polysomy \\
\hline 46 & Sorafenib & oropharynx & $\begin{array}{c}\text { Untreated } \\
\text { primary }\end{array}$ & Incon. & 6.22 & $16+$ & + & Low & Low trisomy \\
\hline 47 & Sorafenib & $\operatorname{larynx}$ & $\begin{array}{l}\text { Local } \\
\text { recurrence }\end{array}$ & - & 2.28 & - & + & High & High polysomy \\
\hline 48 & Sorafenib & oropharynx & $\begin{array}{l}\text { Untreated } \\
\text { primary }\end{array}$ & - & 0.64 & - & - & High & Low polysomy \\
\hline 49 & Sorafenib & oropharynx & $\begin{array}{l}\text { Local } \\
\text { recurrence }\end{array}$ & - & 1.4 & - & - & High & High polysomy \\
\hline 50 & Sorafenib & oropharynx & $\begin{array}{l}\text { Local } \\
\text { recurrence }\end{array}$ & - & 1.3 & - & - & High & High polysomy \\
\hline
\end{tabular}


Table 3 EGFRvIII mutation negative detected by RT-PCR $(\mathbf{n}=19)$ and inconclusive cases $(\mathbf{n}=12)$ (Continued)

\begin{tabular}{|c|c|c|c|c|c|c|c|c|c|}
\hline 51 & Sorafenib & oropharynx & $\begin{array}{l}\text { Local } \\
\text { recurrence }\end{array}$ & - & 1.76 & - & + & Low & $\begin{array}{c}\text { Monosomy/ } \\
\text { Disomy }\end{array}$ \\
\hline 52 & Ispinesib & oropharynx & $\begin{array}{l}\text { Untreated } \\
\text { lymph node }\end{array}$ & Incon. & $\mathrm{NE}$ & - & - & High & Low polysomy \\
\hline 53 & Ispinesib & oropharynx & $\begin{array}{l}\text { Node } \\
\text { recurrence }\end{array}$ & - & 4.05 & $16+$ & - & Low & Amplification \\
\hline
\end{tabular}

Abbreviations: +, positive; -, negative; Incon., inconclusive; NE, not evaluable.

in R/M SCCHN, and may play a role in prognosis. We identified EGFRvIII mutation in $42 \%$ of $53 \mathrm{R} / \mathrm{M} \mathrm{SCCHN}$ tumors. This is in keeping with the first description of EGFRvIII expression by IHC and RT-PCR in $42 \%$ of 33 SCCHN tumors sampled [15]. In vitro studies suggest that EGFRvIII mutated SCCHN cell lines are resistant to the anti-EGFR monoclonal antibody cetuximab [15]. In this study, EGFRvIII was not associated with an inferior response to erlotinib therapy. Importantly, we observed a significant association between the presence of EGFRvIII (mean fold change and copy number by RT-PCR) with greater disease control, regardless of treatment with erlotinib, suggesting that perhaps EGFRvIII may have a prognostic role.

The prognostic or predictive significance of the EGFRvIII mutation in response to systemic therapy in patients with SCCHN has not been previously described. The potential prognostic role of EGFRvIII appears to be independent of any clinicopathologic characteristics. This is consistent with another study where EGFRvIII detected by IHC in 234 of 681 locally advanced SCCHN tumors $(34 \%)$ was associated with increased tumor size but not stage or other clinical factors [36]. In our study, EGFRvIII was not associated with overall survival or
TTP. To our knowledge, EGFRvIII has not been linked to survival in SCCHN. EGFRvIII has been described more extensively in glioblastoma where it results in enhanced proliferation and reduced apoptosis effects that are mediated through increased levels of activated Ras [37] and activation of the PI3K pathway [38]. However, the role of EGFRvIII as a prognostic or predictive marker of response to EGFR inhibitors in glioblastoma remains controversial. EGFRvIII and PTEN co-expression was associated with response to EGFR TKI in 26 patients out of a cohort of 49 patients with recurrent glioma and a validation set of 33 patients [39]. EGFRvIII has been reported as a prognostic marker for poorer survival in some studies $[40,41]$, but not in others $[42,43]$. Conflicting results have been attributed to small sample sizes with incomplete clinical data and varying methods to detect EGFRvIII.

The presence of activating mutations conferring a better prognosis has been reported with EGFR mutations in non-small cell lung cancer (NSCLC) [44] and with PIK3CA mutations in breast cancer [45]. Somatic activating mutations (exon 19 deletion and 21 point mutation) in the EGFR tyrosine kinase domain confer sensitivity to EGFR inhibitors in NSCLC. Patients with

Table 4 Presence of the EGFRvIII mutation is not significantly associated with any clinical characteristics

\begin{tabular}{llll}
\hline Clinical Characteristic & EGFRvlll absent $(\mathbf{n}=\mathbf{1 9 )}$ & EGFRvIll present $(\mathbf{n}=\mathbf{2 2})$ & p-value \\
\hline Male - no., (\%) & $16(84 \%)$ & $18(82 \%)$ & 0.839 (Fisher's) \\
\hline Age - mean, (+/-SD) & $53.5(+/-11.6)$ & $55.1(+/-14.1)$ & 0.685 (t-test) \\
\hline Oropharynx - no., (\%) & $8(42 \%)$ & $6(27 \%)$ & 0.318 (Chi-square) \\
\hline Larynx - no., (\%) & $6(32 \%)$ & $6(27 \%)$ & 0.763 (Chi-square) \\
\hline Oral Cavity - no., (\%) & $2(11 \%)$ & $5(23 \%)$ & 0.271 (Fisher's) \\
\hline Distant metastasis - no., (\%) & $4(21 \%)$ & $12(55 \%)$ & 0.053 (Fisher's) \\
\hline Locoregional recurrence - no., (\%) & $18(95 \%)$ & $16(73 \%)$ & 0.099 (Fisher's) \\
\hline Well-moderately differentiated - no., (\%) & $15(79 \%)$ & $14(64 \%)$ & 0.325 (Fisher's) \\
\hline Poorly differentiated - no., (\%) & $4(21 \%)$ & $8(36 \%)$ & 0.476 (Chi-square) \\
\hline Prior chemotherapy - no., (\%) & $9(47 \%)$ & $8(36 \%)$ & 0.209 (Fisher's) \\
\hline Prior radiotherapy - no., (\%) & $17(89 \%)$ & $22(100 \%)$ & 0.419 (Fisher's) \\
\hline Prior surgery - no., (\%) & $17(89 \%)$ & $17(77 \%)$ & 0.562 (Fisher's) \\
\hline Caucasian - no., (\%) & $15(79 \%)$ & $18(82 \%)$ & 0.735 (Chi-square) \\
\hline Erlotinib treatment - no., (\%) & $12(63 \%)$ & 7 (32\%) & \\
\hline No erlotinib treatment - no., (\%) & $7(38 \%)$ & $15 \%)$ & \\
\hline
\end{tabular}


Table 5 EGFRvIII mutation is associated with disease control

\begin{tabular}{|c|c|c|c|}
\hline \multirow[t]{2}{*}{ EGFRvIII } & \multicolumn{3}{|l|}{ Best Response } \\
\hline & Progressive Disease & Disease Control (Partial Response or Stable Disease) & \\
\hline EGFRvill absent by $\Delta \Delta C t$ & $12(67 \%)$ & $6(33 \%)$ & $P=0.0099$ \\
\hline EGFRvIll present by $\Delta \Delta C t$ & $5(25 \%)$ & $15(75 \%)$ & (Chi-square) \\
\hline EGFRvIll mean fold change & 10.62 & 63.76 & $P=0.04$ \\
\hline EGFRvIll median fold change & 3.16 & 11.11 & (Wilcoxon) \\
\hline \multicolumn{4}{|l|}{ Erlotinib treated patients } \\
\hline EGFRvIll absent by $\triangle \Delta C t$ & $6(55 \%)$ & $5(45 \%)$ & $P=0.21$ \\
\hline EGFRvIll present by $\Delta \Delta \mathrm{Ct}$ & $3(33 \%)$ & $10(77 \%)$ & (Fisher's) \\
\hline \multicolumn{4}{|l|}{ Non-erlotinib treated patients } \\
\hline EGFRvIll absent by $\triangle \triangle C t$ & $6(86 \%)$ & $1(14 \%)$ & $P=0.21$ \\
\hline EGFRvill present by $\Delta \Delta C t$ & $2(29 \%)$ & $5(71 \%)$ & (Fisher's) \\
\hline
\end{tabular}

these mutations also had improved survival and response to chemotherapy alone [46] or placebo [47]. This suggests that EGFR mutations in NSCLC are a good prognostic factor independent of EGFR TKI, hence it may be more difficult to demonstrate the value of EGFR mutations as predictors of benefit to EGFR TKI [44]. The prognostic value of EGFRvIII in SCCHN needs to be verified, and its role as a predictive marker of response to EGFR inhibitor should remain a relevant therapeutic question.

In this study, the prevalence of HPV, p16 and c-MET expression (38\%, 32\% and $63 \%$ respectively) was in keeping with the literature. We did not observe HPV, p16 and c-MET expression to be predictive of disease control, TTP or OS. This may be due to limitations of a small sample size. Consistent with prior reports [21], HPV-16 was the most common HPV subtype in our study. c-MET is a poor prognostic marker in OSCC [48], however the small proportion (11\%) of OSCC in our study precludes any meaningful association.

Limitations of this study include its small sample size, potential bias towards patients with available tumor specimens (larger tumor size), potentially variable fixation and quality of the archival tissues and

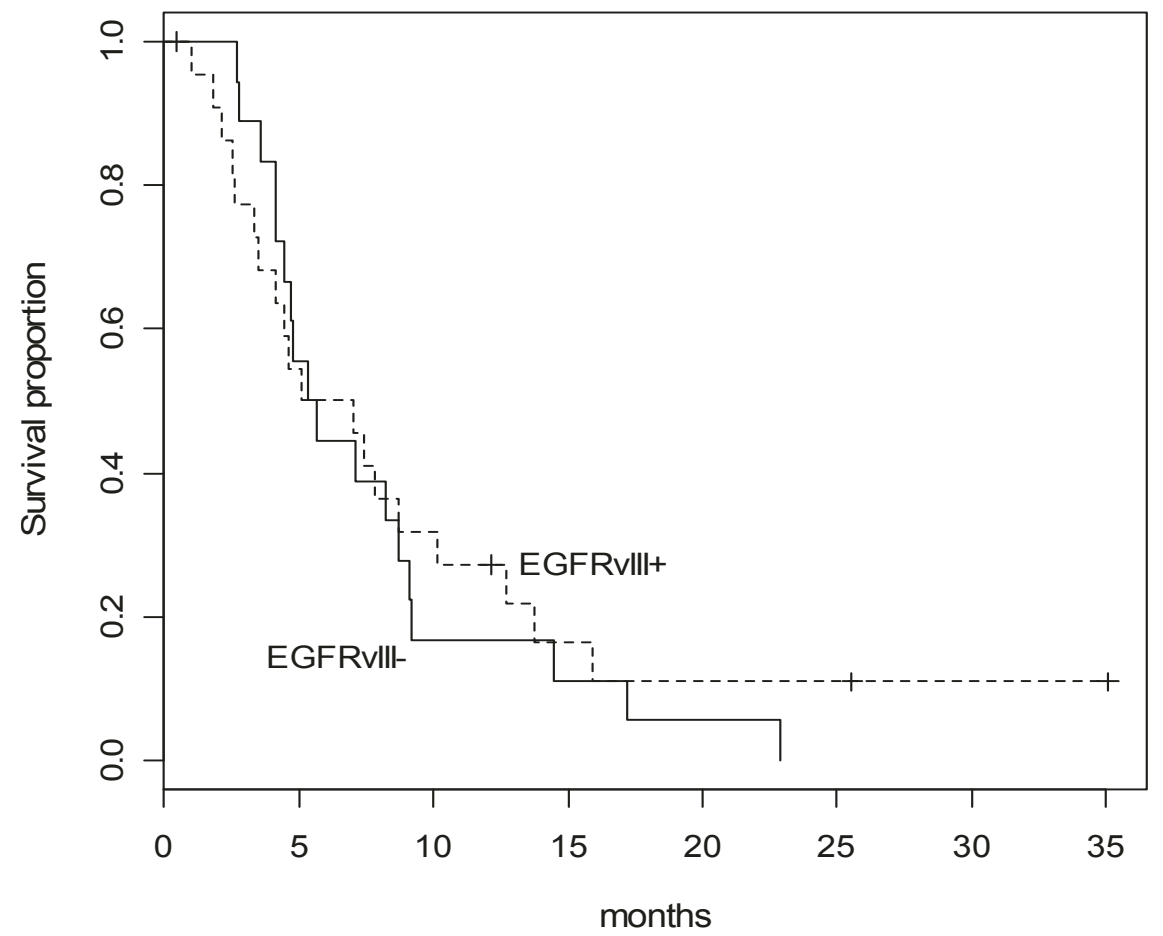

Figure 1 Overall survival by EGFRvIll mutation status (HR $=0.91,95 \% \mathrm{Cl}: 0.32-2.60, \mathrm{p}=0.85$ ). 
Table 6 HPV by PCR, p16 by IHC, c-MET by IHC are not associated with disease control

\begin{tabular}{|c|c|c|c|}
\hline & \multicolumn{3}{|c|}{ Best Response } \\
\hline & $\begin{array}{l}\text { Progressive } \\
\text { Disease }\end{array}$ & $\begin{array}{l}\text { Disease Control } \\
\text { (Partial Response or } \\
\text { Stable Disease) }\end{array}$ & \\
\hline $\begin{array}{l}\text { HPV PCR } \\
\text { negative }\end{array}$ & $14(50 \%)$ & $14(50 \%)$ & $P=0.86$ \\
\hline $\begin{array}{l}\text { HPV PCR } \\
\text { positive }\end{array}$ & $9(47 \%)$ & $10(53 \%)$ & (Chi-square) \\
\hline $\begin{array}{l}\text { P16 IHC } \\
\text { negative }\end{array}$ & $14(47 \%)$ & 16 (53\%) & $P=0.67$ \\
\hline $\begin{array}{l}\text { P16 IHC } \\
\text { positive }\end{array}$ & $8(53 \%)$ & $7(47 \%)$ & (Chi-square) \\
\hline $\begin{array}{l}\text { c-MET IHC } \\
<\text { or }=2\end{array}$ & $9(56 \%)$ & 7 (44\%) & $P=0.39$ \\
\hline C-MET IHC >2 & $12(43 \%)$ & $16(57 \%)$ & (Chi-square) \\
\hline
\end{tabular}

potential variation in marker status of primary tumor compared with recurrent or metastatic tumors (to our knowledge, this is theoretical and has not been described). Due to the absence of an untreated control group in this study ('control' patients received sorafenib or ispinesib), our results cannot conclusively confirm the prognostic versus predictive value of a biomarker. Although our methods did not use an antibody for EGFRvIII detection, we acknowledge that the use of RT-PCR in FFPE samples has demonstrated superior accuracy relative to IHC tests [49] and may allow greater applicability to settings where frozen tissue is unavailable.

\section{Conclusion}

Predictors of response to EGFR inhibitors in SCCHN remain elusive. Biomarkers are desperately needed to guide patient selection in SCCHN. EGFRvIII remains an interesting tumor-specific target worthy of further exploration as a prognostic or predictive marker of response to EGFR inhibitor therapy in SCCHN. Larger prospective randomized studies are required to distinguish the prognostic and predictive significance of EGFRvIII, HPV, p16, c-MET and EGFR GCN in SCCHN treated with EGFR inhibitors.

Table 7 Concordance of p16 IHC status with HPV by PCR and HPV by ISH

\begin{tabular}{llll}
\hline & $\begin{array}{l}\text { P16 IHC } \\
\text { positive }\end{array}$ & $\begin{array}{l}\text { P16 IHC } \\
\text { negative }\end{array}$ & \\
\hline HPV PCR positive & 10 & 5 & $P=0.0016$ \\
$\begin{array}{l}\text { HPV PCR } \\
\text { negative }\end{array}$ & 5 & 25 & $\begin{array}{l}\text { (Chi- } \\
\text { Square) }\end{array}$ \\
\hline HPV ISH positive & 6 & & $P=0.0002$ \\
\hline HPV ISH negative & 7 & 0 & (Fisher's) \\
\hline
\end{tabular}

\section{Acknowledgements}

This work was supported by an unrestricted grant from Pfizer Canada, Inc.

\section{Author details}

'Division of Medical Oncology and Hematology, Princess Margaret Hospital, University Health Network, Toronto, Ontario, Canada. ${ }^{2}$ Department of Pathology, Princess Margaret Hospital, University Health Network, Toronto, Ontario, Canada. ${ }^{3}$ Advanced Molecular Profiling Laboratory, Princess Margaret Hospital, University Health Network, Toronto, Ontario, Canada. ${ }^{4}$ Department of Biostatistics; Princess Margaret Hospital, University Health Network, Toronto, Ontario, Canada.

\section{Authors' contributions}

NC participated in the study design, data acquisition, immunohistochemical interpretation and drafted the manuscript. BP-O participated in the histological examination, immunohistochemical interpretation and manuscript preparation. KZ, NAP, JH, TZ, M-ST, SK-R carried out immunostaining, ISH, PCR analysis, and prepared and reviewed the manuscript. OL performed the FISH analysis and reviewed the manuscript. LW performed the statistical analysis. EC participated in patient management and reviewed the manuscript. LS conceived of the study, participated in its design and coordination, and prepared and revised the manuscript. All authors read and approved the final manuscript.

\section{Competing interests}

The authors declare that they have no competing interests.

Received: 31 December 2010 Accepted: 27 February 2011

Published: 27 February 2011

\section{References}

1. Grandis JR, Tweardy DJ: Elevated levels of transforming growth factor alpha and epidermal growth factor receptor messenger RNA are early markers of carcinogenesis in head and neck cancer. Cancer Res 1993, 53:3579-3584.

2. Woodburn JR: The epidermal growth factor receptor and its inhibition in cancer therapy. Pharmacol Ther 1999, 82:241-250.

3. Temam S, Kawaguchi H, El-Naggar AK, Jelinek J, Tang H, Liu DD, Lang W, Issa JP, Lee JJ, Mao L: Epidermal growth factor receptor copy number alterations correlate with poor clinical outcome in patients with head and neck squamous cancer. J Clin Oncol 2007, 25:2164-2170.

4. Chung CH, Ely K, McGavran L, Varella-Garcia M, Parker J, Parker N, Jarrett C, Carter J, Murphy BA, Netterville J, et al: Increased epidermal growth factor receptor gene copy number is associated with poor prognosis in head and neck squamous cell carcinomas. J Clin Oncol 2006, 24:4170-4176.

5. Bonner JA, Harari PM, Giralt J, Azarnia N, Shin DM, Cohen RB, Jones CU, Sur R, Raben D, Jassem J, et al: Radiotherapy plus cetuximab for squamous-cell carcinoma of the head and neck. NEnglJ Med 2006 354:567-578

6. Cohen EE, Rosen F, Stadler WM, Recant W, Stenson K, Huo D, Vokes EE: Phase II trial of ZD1839 in recurrent or metastatic squamous cell carcinoma of the head and neck. J Clin Oncol 2003, 21:1980-1987.

7. Siu LL, Soulieres D, Chen EX, Pond GR, Chin SF, Francis P, Harvey L, Klein M, Zhang W, Dancey J, et al: Phase I/II trial of erlotinib and cisplatin in patients with recurrent or metastatic squamous cell carcinoma of the head and neck: a Princess Margaret Hospital phase II consortium and National Cancer Institute of Canada Clinical Trials Group Study. J Clin Oncol 2007, 25:2178-2183.

8. Soulieres D, Senzer NN, Vokes EE, Hidalgo M, Agarwala SS, Siu LL: Multicenter phase II study of erlotinib, an oral epidermal growth factor receptor tyrosine kinase inhibitor, in patients with recurrent or metastatic squamous cell cancer of the head and neck. J Clin Oncol 2004, 22:77-85.

9. Burtness B, Goldwasser MA, Flood W, Mattar B, Forastiere AA: Phase II randomized trial of cisplatin plus placebo compared with cisplatin plus cetuximab in metastatic/recurrent head and neck cancer: an Eastern Cooperative Oncology Group study. J Clin Oncol 2005, 23:8646-8654.

10. Vermorken JB, Mesia R, Rivera F, Remenar E, Kawecki A, Rottey S, Erfan J, Zabolotnyy D, Kienzer HR, Cupissol D, et al: Platinum-based chemotherapy plus cetuximab in head and neck cancer. N Engl I Med 2008, 359:1116-1127. 
11. Grandal MV, Zandi R, Pedersen MW, Willumsen BM, van DB, Poulsen HS: EGFRvIll escapes down-regulation due to impaired internalization and sorting to lysosomes. Carcinogenesis 2007, 28:1408-1417.

12. Huang HS, Nagane M, Klingbeil CK, Lin H, Nishikawa R, Ji XD, Huang CM, Gill GN, Wiley HS, Cavenee WK: The enhanced tumorigenic activity of a mutant epidermal growth factor receptor common in human cancers is mediated by threshold levels of constitutive tyrosine phosphorylation and unattenuated signaling. J Biol Chem 1997, 272:2927-2935.

13. Nishikawa R, Ji XD, Harmon RC, Lazar CS, Gill GN, Cavenee WK, Huang HJ: A mutant epidermal growth factor receptor common in human glioma confers enhanced tumorigenicity. Proc Natl Acad Sci USA 1994, 91:7727-7731

14. Ji H, Zhao X, Yuza Y, Shimamura T, Li D, Protopopov A, Jung BL, McNamara K, Xia H, Glatt KA, et al: Epidermal growth factor receptor variant III mutations in lung tumorigenesis and sensitivity to tyrosine kinase inhibitors. Proc Natl Acad Sci USA 2006, 103:7817-7822.

15. Sok JC, Coppelli FM, Thomas SM, Lango MN, Xi S, Hunt JL, Freilino ML, Graner MW, Wikstrand CJ, Bigner DD, et al: Mutant epidermal growth factor receptor (EGFRvIII) contributes to head and neck cancer growth and resistance to EGFR targeting. Clin Cancer Res 2006, 12:5064-5073.

16. Gillison ML, Koch WM, Capone RB, Spafford M, Westra WH, Wu L, Zahurak ML, Daniel RW, Viglione M, Symer DE, et al: Evidence for a causal association between human papillomavirus and a subset of head and neck cancers. J Natl Cancer Inst 2000, 92:709-720.

17. Shi W, Kato H, Perez-Ordonez B, Pintilie M, Huang S, Hui A, O'Sullivan B, Waldron J, Cummings B, Kim J, et al: Comparative prognostic value of HPV16 E6 mRNA compared with in situ hybridization for human oropharyngeal squamous carcinoma. J Clin Oncol 2009, 27:6213-6221.

18. Gillison ML, D'Souza G, Westra W, Sugar E, Xiao W, Begum S, Viscidi R: Distinct risk factor profiles for human papillomavirus type 16-positive and human papillomavirus type 16-negative head and neck cancers. J Natl Cancer Inst 2008, 100:407-420.

19. Westra WH, Taube JM, Poeta ML, Begum S, Sidransky D, Koch WM: Inverse relationship between human papillomavirus-16 infection and disruptive p53 gene mutations in squamous cell carcinoma of the head and neck. Clin Cancer Res 2008, 14:366-369.

20. Weinberger PM, Yu Z, Haffty BG, Kowalski D, Harigopal M, Brandsma J, Sasaki C, Joe J, Camp RL, Rimm DL, Psyrri A: Molecular classification identifies a subset of human papillomavirus-associated oropharyngeal cancers with favorable prognosis. J Clin Oncol 2006, 24:736-747.

21. Fakhry C, Westra WH, Li S, Cmelak A, Ridge JA, Pinto H, Forastiere A, Gillison ML: Improved survival of patients with human papillomaviruspositive head and neck squamous cell carcinoma in a prospective clinical trial. J Natl Cancer Inst 2008, 100:261-269.

22. Kumar B, Cordell KG, Lee JS, Worden FP, Prince ME, Tran HH, Wolf GT, Urba SG, Chepeha DB, Teknos TN, et al: EGFR, p16, HPV Titer, Bcl-xL and p53, sex, and smoking as indicators of response to therapy and survival in oropharyngeal cancer. J Clin Oncol 2008, 26:3128-3137.

23. Lassen P, Eriksen JG, Hamilton-Dutoit S, Tramm T, Alsner J, Overgaard J: Effect of HPV-associated p16INK4A expression on response to radiotherapy and survival in squamous cell carcinoma of the head and neck. J Clin Oncol 2009, 27:1992-1998.

24. Smith EM, Wang D, Kim Y, Rubenstein LM, Lee JH, Haugen TH, Turek LP. P16INK4a expression, human papillomavirus, and survival in head and neck cancer. Oral Oncol 2008, 44:133-142.

25. Knowles LM, Stabile LP, Egloff AM, Rothstein ME, Thomas SM, Gubish CT, Lerner EC, Seethala RR, Suzuki S, Quesnelle KM, et al: HGF and c-Met participate in paracrine tumorigenic pathways in head and neck squamous cell cancer. Clinical Cancer Research 2009, 15:3740-3750.

26. Chen YS, Wang JT, Chang YF, Liu BY, Wang YP, Sun A, Chiang CP: Expression of hepatocyte growth factor and c-met protein is significantly associated with the progression of oral squamous cell carcinoma in Taiwan. Journal of Oral Pathology \& Medicine 2004, 33:209-217.

27. Allen C, Duffy S, Teknos T, Islam M, Chen Z, Albert PS, Wolf G, Van WC: Nuclear factor-kappaB-related serum factors as longitudinal biomarkers of response and survival in advanced oropharyngeal carcinoma. Clin Cancer Res 2007, 13:3182-3190.

28. Di Renzo MF, Olivero M, Martone T, Maffe A, Maggiora P, Stefani AD, Valente G, Giordano S, Cortesina G, Comoglio PM: Somatic mutations of the MET oncogene are selected during metastatic spread of human HNSC carcinomas. Oncogene 2000, 19:1547-1555.

29. Wheeler DL, Huang S, Kruser TJ, Nechrebecki MM, Armstrong EA, Benavente S, Gondi V, Hsu KT, Harari PM: Mechanisms of acquired resistance to cetuximab: role of HER (ErbB) family members. Oncogene 2008, 27:3944-3956.

30. Tang PA, Siu LL, Chen EX, Hotte SJ, Chia S, Schwarz JK, Pond GR, Johnson C, Colevas AD, Synold TW, et al: Phase II study of ispinesib in recurrent or metastatic squamous cell carcinoma of the head and neck. Invest New Drugs 2008, 26:257-264.

31. Elser $C$, Siu LL, Winquist $E$, Agulnik M, Pond GR, Chin SF, Francis $P$, Cheiken R, Elting J, McNabola A, et al: Phase II trial of sorafenib in patients with recurrent or metastatic squamous cell carcinoma of the head and neck or nasopharyngeal carcinoma. J Clin Oncol 2007, 25:3766-3773.

32. Weinberger PM, Yu Z, Haffty BG, Kowalski D, Harigopal M, Sasaki C, Rimm DL, Psyrri A: Prognostic significance of p16 protein levels in oropharyngeal squamous cell cancer. Clin Cancer Res 2004, 10:5684-5691.

33. Agulnik M, da Cunha SG, Hedley D, Nicklee T, Dos Reis PP, Ho J, Pond GR, Chen $H$, Chen S, Shyr $Y$, et al: Predictive and pharmacodynamic biomarker studies in tumor and skin tissue samples of patients with recurrent or metastatic squamous cell carcinoma of the head and neck treated with erlotinib. Journal of Clinical Oncology 2007, 25:2184-2190.

34. Tsao MS, Sakurada A, Cutz JC, Zhu CQ, Kamel-Reid S, Squire J, Lorimer I, Zhang T, Liu N, Daneshmand $M$, et al: Erlotinib in lung cancer - molecular and clinical predictors of outcome. N Engl J Med 2005, 353:133-144.

35. Cappuzzo F, Hirsch FR, Rossi E, Bartolini S, Ceresoli GL, Bemis L, Haney J, Witta S, Danenberg K, Domenichini I, et al: Epidermal growth factor receptor gene and protein and gefitinib sensitivity in non-small-cell lung cancer. J Natl Cancer Inst 2005, 97:643-655.

36. Eriksen JGLP, Overgaard J: The EGFRvill variant in squamous cell carcinomas of the head and neck: Expression and correlation with clinico-pathological parameters in 681 patients from the randomised DAHANCA 6/7 study. ECCO 15 - 34th ESMO Multidisciplinary Congress Berlin; 2009, 472, September 2009

37. Prigent SA, Nagane M, Lin H, Huvar I, Boss GR, Feramisco JR, Cavenee WK, Huang HS: Enhanced tumorigenic behavior of glioblastoma cells expressing a truncated epidermal growth factor receptor is mediated through the Ras-Shc-Grb2 pathway. J Biol Chem 1996, 271:25639-25645.

38. Huang PH, Mukasa A, Bonavia R, Flynn RA, Brewer ZE, Cavenee WK, Furnari FB, White FM: Quantitative analysis of EGFRvIll cellular signaling networks reveals a combinatorial therapeutic strategy for glioblastoma. Proc Natl Acad Sci USA 2007, 104:12867-12872.

39. Mellinghoff IK, Wang MY, Vivanco I, Haas-Kogan DA, Zhu S, Dia EQ, Lu KV, Yoshimoto K, Huang JH, Chute DJ, et al: Molecular determinants of the response of glioblastomas to EGFR kinase inhibitors. N Engl J Med 2005, 353:2012-2024.

40. Pelloski CE, Ballman KV, Furth AF, Zhang L, Lin E, Sulman EP, Bhat K, McDonald JM, Yung WK, Colman $H$, et al: Epidermal growth factor receptor variant III status defines clinically distinct subtypes of glioblastoma. J Clin Oncol 2007, 25:2288-2294

41. Shinojima N, Tada K, Shiraishi S, Kamiryo T, Kochi M, Nakamura H, Makino K, Saya $\mathrm{H}$, Hirano $\mathrm{H}$, Kuratsu J, et al: Prognostic value of epidermal growth factor receptor in patients with glioblastoma multiforme. Cancer Res 2003, 63:6962-6970.

42. Heimberger AB, Hlatky R, Suki D, Yang D, Weinberg J, Gilbert M, Sawaya R, Aldape K: Prognostic effect of epidermal growth factor receptor and EGFRvIII in glioblastoma multiforme patients. Clin Cancer Res 2005, 11:1462-1466

43. Liu L, Backlund LM, Nilsson BR, Grander D, Ichimura K, Goike HM, Collins VP: Clinical significance of EGFR amplification and the aberrant EGFRvIll transcript in conventionally treated astrocytic gliomas. J Mol Med 2005, 83:917-926.

44. Eberhard DA, Giaccone G, Johnson BE: Biomarkers of response to epidermal growth factor receptor inhibitors in Non-Small-Cell Lung Cancer Working Group: standardization for use in the clinical trial setting. J Clin Oncol 2008, 26:983-994.

45. Kalinsky K, Jacks LM, Heguy A, Patil S, Drobnjak M, Bhanot UK, Hedvat CV, Traina TA, Solit D, Gerald W, Moynahan ME: PIK3CA mutation associates with improved outcome in breast cancer. Clin Cancer Res 2009, 15:5049-5059. 
46. Eberhard DA, Johnson BE, Amler LC, Goddard AD, Heldens SL, Herbst RS, Ince WL, Janne PA, Januario T, Johnson DH, et al: Mutations in the epidermal growth factor receptor and in KRAS are predictive and prognostic indicators in patients with non-small-cell lung cancer treated with chemotherapy alone and in combination with erlotinib. I Clin Oncol 2005, 23:5900-5909.

47. Shepherd FA, Tsao MS: Unraveling the mystery of prognostic and predictive factors in epidermal growth factor receptor therapy. $J$ Clin Oncol 2006, 24:1219-1220, author reply 1220-1211.

48. Lo ML, Leonardi R, Mignogna MD, Pannone G, Rubini C, Pieramici T, Trevisiol L, Ferrari F, Serpico R, Testa N, et al: Scatter factor receptor (cMet) as possible prognostic factor in patients with oral squamous cell carcinoma. Anticancer Research 2004, 24:1063-1069.

49. Yoshimoto K, Dang J, Zhu S, Nathanson D, Huang T, Dumont R, Seligson DB, Yong WH, Xiong Z, Rao N, et al: Development of a real-time RT-PCR assay for detecting EGFRvill in glioblastoma samples. Clin Cancer Res 2008, 14:488-493.

doi:10.1186/1758-3284-3-11

Cite this article as: Chau et al:: The association between EGFR variant III, HPV, p16, c-MET, EGFR gene copy number and response to EGFR inhibitors in patients with recurrent or metastatic squamous cell carcinoma of the head and neck. Head \& Neck Oncology 2011 3:11.

\section{Submit your next manuscript to BioMed Central} and take full advantage of:

- Convenient online submission

- Thorough peer review

- No space constraints or color figure charges

- Immediate publication on acceptance

- Inclusion in PubMed, CAS, Scopus and Google Scholar

- Research which is freely available for redistribution

Submit your manuscript at www.biomedcentral.com/submit 